\title{
Detection of Stochastic Signals in the Frequency Domain
}

\author{
Y.T. Chan, H.C. So, Q. Yuan, R. Inkol
}

\begin{abstract}
Signal detection and processing are now mostly frequency domain operations due to the easy availability of FFT chips. There is an optimal signal detector in the continuous frequency domain. Optimality here refers to a detector that has a maximum deflection ratio (DR) and its realization requires that the signal power spectral density (PSD) be known. Implementation of this detector, by necessity, is through a discretization of its continuous version. This discretization is only an approximation to the theoretical and suffers a loss in detection performance which becomes significant at high signal to noise ratios. To avoid this loss, this paper derives, from first principles, an optimal detector in the discrete frequency domain. When the signal PSD is not know $n$, this detector takes the simple form of using the peak of the data PSD estimate as the test statistic. The segment length used in the Bartlett spectral estimator determines the detector's DR. The segment length that maximizes the DR is inversely proportional to the signal bandwidth. Thus an optimal detector is realizable from just the knowledge of the signal bandwidth, which is normally available in practice. Examples and simulation results are provided to illustrate the new detector's properties and performance.
\end{abstract}

\section{INTRODUCTION}

$\mathrm{I}_{\mathrm{r}}^{\mathrm{N}}$ $\mathrm{N}$ many applications, there is a need to detect the occurrence of a signal in the presence of noise. Examples are the detection of a spread spectrum, radar and sonar signals. Due to the ease of hardware implementation, the detector invariably uses a fast Fourier transform (FFT) processor and computes a test statistic in the frequency domain for detection. Let the received data be

$$
y(n)= \begin{cases}s(n)+\phi(n), & \text { signal present } \\ \phi(n), & \text { no signal present }\end{cases}
$$

where $s(n)$ is a stochastic signal independent of the background zero mean Gaussian white noise sequence $\phi(n)$. The problem is to decide, from $y(n), n=0, \ldots, N-1$, whether $s(n)$ is present.

A standard FFT detector computes

$$
\Lambda(m)=|Y(m)|^{2}
$$

where

$$
Y(m)=\sum_{n=0}^{N-1} y(n) e^{-j \frac{2 \pi m n}{N}}
$$

is the discrete Fourier transform of $y(n)$. It then selects the largest value of $\Lambda\left(m^{*}\right)$ and compares it against a threshold TH. A detection occurs when $\Lambda\left(m^{*}\right) \geq \mathrm{TH}$. This detector is optimum if $s(n)$ is a sinusoid of unknown amplitude,

Y.T. Chan and Q. Yuan are with the Department of Electrical and Computer Engineering, Royal Military College of Canada, Kingston, Ontario, Canada K7K 5L0. H.C. So is with the Department of Electronic Engineering, City University of Hong Kong, Hong Kong. R. Inkol is with the Defence Research Establishment Ottawa, Department of National Defence, Ottawa, Ontario, Canada K1A 0Z4 phase and frequency [1], and if the frequency is equal to a bin frequency, i.e., it equals $\frac{2 \pi m^{*}}{N}, m^{*}=$ integer. Optimum detection here means that for a given probability of false alarm $\left(P_{F A}\right)$, the detector gives the maximum probability of detection $\left(P_{D}\right)$.

Another common detector uses the test statistic

$$
\Lambda_{T}=\sum_{m=0}^{N-1}|Y(m)|^{2}=\sum_{n=0}^{N-1} y^{2}(n)
$$

which takes on the various names of radiometers, energy detector and quadratic detector [2]. It is optimum if $s(n)$ is a Gaussian bandlimited white noise process. Both [1] and [2] have considered another criterion for optimality, which is the maximization of the deflection ratio (DR)

$$
\mathrm{DR}=\frac{[E\{\Lambda / 1\}-E\{\Lambda / 0\}]^{2}}{V A R\{\Lambda / 0\}}
$$

In (5) $E\{$.$\} denotes the expected value, V A R\{$.$\} the vari-$ ance, and $\Lambda / 1$ the value of the test statistic when a signal is present, and $\Lambda / 0$ when not. The $\mathrm{DR}$, which is a signal to noise parameter, is an intuitively appealing measure since its maximization will also maximize the separation between the means of $\Lambda / 1$ and $\Lambda / 0$, normalized by the variance of $\Lambda / 0$. It also leads to tractable solutions for detectors [3]. However, a detector that provides a maximum DR is not necessarily the one that provides maximum $P_{D}$ for a fixed $P_{F A}[3]$.

The detector that maximizes the DR is [1] [2]

$$
\Lambda_{c}=\frac{1}{2 \pi} \int_{-\pi}^{\pi}|Y(\omega)|^{2} G_{s s}(\omega) d \omega
$$

where

$$
Y(\omega)=\sum_{n=0}^{N-1} y(n) e^{-j \omega n}
$$

and $G_{s s}(\omega)$ is the true (assumed known) power spectral density (PSD) of $s(n)$. It is assumed, without loss of generality, that $G_{s s}(\omega)$ is bandlimited between $-\pi$ and $\pi$. The interpretation of (6) is that the optimal detector computes the periodogram of the data and matches it against $G_{s s}(\omega)$. Thus in a sense it is a matched filter in the frequency domain. For digital implementation, it is necessary to discretize (6) to

$$
\Lambda_{d}=\sum_{m=0}^{N-1}|Y(m)|^{2} G_{s s}\left(\frac{2 \pi m}{N}\right)
$$


which will suffer a loss in optimality because of the approximation due to discretization.

Since the FFT has available only discrete frequency components, it is important to design an optimal detector for the discrete case. Such a detector is presently not available and its derivation is the subject of this paper. The organization of the paper is as follows. Section II first gives the development of the optimal discrete detector, which is different from the one in (8). It then moves on to study the properties of the new detector and compares them against those of (2) and (8). Section III compares examples of the new detector, versus the existing ones. The conclusions are in section IV.

\section{THE OPTIMAL DISCRETE DETECTOR}

\section{II.1 Derivation}

From $y(n), n=0, \ldots, N-1$, the new test statistic is

$$
\Lambda=\sum_{m=0}^{L-1} W(m) \widetilde{G}_{y y}(m)
$$

where

$$
\begin{gathered}
\widetilde{G}_{y y}(m)=\frac{1}{K} \sum_{k=0}^{K-1}\left|Y_{k}(m)\right|^{2} \\
Y_{k}(m)=\sum_{n=k L}^{(k+1) L-1} y(n) e^{-j \frac{2 \pi m n}{L}}, \quad K L=N
\end{gathered}
$$

and $W(m)$ is a spectral weighting sequence. Thus (9) weights the Bartlett [4] spectral estimate with $W(m)$ and sums the products to form $\Lambda_{o}$. The problem is to select $W(m)$ and $L$, the number of data points in a Bartlett segment, to maximize the DR of (5).

Let

$$
S_{k}(m)=\sum_{n=k L}^{(k+1) L-1} s(n) e^{-j \frac{2 \pi m n}{L}}
$$

and

$$
\Phi_{k}(m)=\sum_{n=k L}^{(k+1) L-1} \phi(n) e^{-j \frac{2 \pi m n}{L}}
$$

Then from (10), when $s(n)$ is present,

$$
\widetilde{G}_{y y}(m)=\frac{1}{K} \sum_{k=0}^{K-1}\left(S_{k}(m)+\Phi_{k}(m)\right)\left(S_{k}^{*}(m)+\Phi_{k}^{*}(m)\right)
$$

with * denoting the complex conjugate. Taking expected value gives

$$
E\left\{\widetilde{G}_{y y}(m)\right\}=\bar{G}_{y y}(m)=\bar{G}_{s s}(m)+\bar{G}_{\phi \phi}(m)
$$

where

$$
\begin{aligned}
\bar{G}_{s s}(m) & =E\left\{\left|S_{k}(m)\right|^{2}\right\} \\
\bar{G}_{\phi \phi}(m) & =E\left\{\left|\Phi_{k}(m)\right|^{2}\right\}
\end{aligned}
$$

and

$$
E\left\{S_{k}(m) \Phi_{k}(m)\right\}=0
$$

Similarly, when there is no signal,

$$
E\left\{\widetilde{G}_{y y}(m)\right\}=E\left\{\widetilde{G}_{\phi \phi}(m)\right\}=\bar{G}_{\phi \phi}(m)
$$

Hence

$$
E\{\Lambda / 1\}-E\{\Lambda / 0\}=\sum_{m=0}^{L-1} W(m) \bar{G}_{s s}(m)
$$

Next, using

$$
V A R\{\Lambda / 0\}=E\left\{(\Lambda / 0)^{2}\right\}-E^{2}\{\Lambda / 0\}
$$

it is easy to verify that

$$
V A R\{\Lambda / 0\}=\sum_{m=0}^{L-1} W^{2}(m)\left[V A R\left\{\widetilde{G}_{\phi \phi}(m)\right\}\right]
$$

From [4], the variance of $\widetilde{G}_{\phi \phi}(m)$ is $\frac{2 L^{3} \sigma_{\phi}^{4}}{N}$, where $\sigma_{\phi}^{2}=$ $E\left\{\phi^{2}(n)\right\}$. Note that the $L^{3}$ term appears in the variance because the Fourier transform in (13) is not divided by $L$. Hence the DR for the detector of (9) is, from (18) and (20)

$$
\mathrm{DR}=N \frac{\left[\sum_{m} W(m) \bar{G}_{s s}(m)\right]^{2}}{2 L^{3} \sigma_{\phi}^{4} \sum_{m} W^{2}(m)}
$$

In (21) and in the sequel, unless otherwise stated, the summation limits are from 0 to $L-1$. Applying the Schwartz's inequality [6] to $(21)$ gives the $W(m)$ that maximizes the DR as

$$
W(m)=\bar{G}_{s s}(m)
$$

Substituting (22) into (9) yields, in the sense of a detector whose DR is a maximum,

$$
\Lambda_{o}=\sum_{m} \bar{G}_{s s}(m) \widetilde{G}_{y y}(m)
$$

with the corresponding

$$
\mathrm{DR}_{\mathrm{o}}=\frac{N \sum_{m} \bar{G}_{s s}^{2}(m)}{2 L^{3} \sigma_{\phi}^{4}}=\frac{N \sum_{m} W^{2}(m)}{2 L^{3} \sigma_{\phi}^{4}}
$$

A further maximization of $\mathrm{DR}_{\mathrm{o}}$ of $(24)$ is available through the selection of $L$, the number of data points in a Bartlett segment. However, in all the examples studied for $\Lambda_{o}$ in section III, $L=N$ always gives the maximum DR. Thus the available extra degree of freedom of selecting $L$ appears not useful for the detector of (23).

The optimum detector in (23) first finds the spectral estimate of the data, and weights it, at each frequency bin, with the expected value of the signal spectral estimate. This expected value is calculated based on the same number of segments and segment length used in the spectral estimate of the data. The detector then sums all the weighted spectral components and compares the sum against a threshold. This detector is different from the detector in (6), or its discrete approximation in (8), which uses the true PSD as weights. 
In practice, the true PSD of $s(n)$ is often not known although some knowledge of spectral features such as bandwidth or location of the PSD peak will be available. It will not be possible to compute the optimum weights $W(m)$ when the true PSD of $s(n)$ is not known. Here it is more appropriate to use the detector

$$
\Lambda_{a}=\widetilde{G}_{y y}(m)
$$

and select the $m$ that gives the highest $\widetilde{G}_{y y}(m)$ and compare it against a threshold. Of course if the peak location of the PSD of $s(n)$ is known a priori to be at $m_{0}$, then $\Lambda_{a}$ simply equals $\widetilde{G}_{y y}\left(m_{0}\right)$. Note that (25) is similar to (2) except that instead of using the periodogram as in (2), (25) uses the Bartlett spectral estimate. The DR of $\Lambda_{a}$ is hence dependent on $L$, the number of points in a Bartlett segment. For $\Lambda_{a}$, unlike $\Lambda_{o}$ of (23), the extra degree of freedom provided by choosing $L$ to maximize the DR is useful. Indeed, the examples in section III for $\Lambda_{a}$ show that the optimum $L$ is roughly inversely proportional to the bandwidth of $s(n)$. This is certainly an important result for designing the detector $\Lambda_{a}$. It gives a guide for selecting the optimum data segment length $L$ for the Bartlett spectral estimate.

\section{II.2 Computation of the DRs}

From (16) and (22)

$$
W(m)=E\left\{S_{k}(m) S_{k}^{*}(m)\right\}
$$

But the expected value of the $k$ th periodogram is independent of $k$, since $s(n)$ is stationary. Hence from (26),

$$
W(m)=\sum_{u} \sum_{v} E\{s(u) s(v)\} e^{-j \frac{2 \pi m}{L}(u-v)}
$$

Let $u-v=i$ and (27) becomes

$$
W(m)=\sum_{u} \sum_{i=-u}^{L-1-u} R_{s s}(i) e^{-j \frac{2 \pi m i}{L}}
$$

where

$$
R_{s s}(i)=E\{s(u) s(v)\}=R_{s s}(u-v)=R_{s s}(-i)
$$

and is the autocorrelation function of $s(n)$. Using (29) in (28) simplifies it, with $\omega_{m}=\frac{2 \pi m}{L}$, to

$$
W(m)=\sum_{i} 2(L-i) R_{s s}(i) \cos \omega_{m} i-L R_{s s}(0)
$$

Squaring (30) yields

$$
\begin{aligned}
& W^{2}(m)=L^{2} R_{s s}^{2}(0) \\
& \quad+\sum_{i} \sum_{k} 4(L-i)(L-k) R_{s s}(i) R_{s s}(k) \cos \omega_{m} i \cos \omega_{m} k \\
& -2 L R_{s s}(0) \sum_{i} 2(L-i) R_{s s}(i) \cos \omega_{m} i
\end{aligned}
$$

Summing (31) over all $m$ gives

$$
\begin{aligned}
& \sum_{m} W^{2}(m)=\sum_{m} L^{2} R_{s s}^{2}(0) \\
& \quad+\sum_{i} \sum_{k} 4(L-i)(L-k) R_{s s}(i) R_{s s}(k) \sum_{m} \cos \omega_{m} i \cos \omega_{m} k \\
& \quad-2 L R_{s s}(0) \sum_{i} 2(L-i) R_{s s}(i) \sum_{m} \cos \omega_{m} i
\end{aligned}
$$

Using the summations

$$
\sum_{m=0}^{L-1} \cos \omega_{m} i \cos \omega_{m} k= \begin{cases}\frac{L}{2,} & \text { for } i=k \neq 0 \\ L, & \text { for } i=k=0 \\ 0, & \text { for } i \neq k\end{cases}
$$

and

$$
\sum_{m=0}^{L-1} \cos \omega_{m} i= \begin{cases}L, & \text { for } i=0 \\ 0, & \text { for } i \neq 0\end{cases}
$$

in (32), and after some simplifications, it results in

$$
\sum_{m} W^{2}(m)=L^{3} R_{s s}^{2}(0)+2 L \sum_{i=1}^{L-1}(L-i)^{2} R_{s s}^{2}(i)
$$

which is a convenient formula for the computation of $\mathrm{DR}_{o}$ in (24).

Next for $\Lambda_{a}$ of (25), its DR is

$$
\mathrm{DR}_{a}=\frac{\left[E\left\{\widetilde{G}_{s s}\left(m_{0}\right)\right\}\right]^{2}}{\operatorname{VAR}\left\{\widetilde{G}_{\phi \phi}(m)\right\}}
$$

which on using $(30)$, since $W(m)$ in (30) equals $E\left\{\widetilde{G}_{s s}(m)\right\}$, gives

$$
\mathrm{DR}_{a}=\frac{N\left[\sum_{i} 2(L-i) R_{s s}(i) \cos \omega_{0} i-L R_{s s}(0)\right]^{2}}{2 L^{3} \sigma_{\phi}^{4}}
$$

where $\omega_{0}=\frac{2 \pi m_{0}}{L}$.

Finally, for $\Lambda_{d}$ of (8), the weights are

$$
W(m)=G_{s s}\left(\frac{2 \pi m}{N}\right) \approx \sum_{i=1}^{p-1} 2 R_{s s}(i) \cos \omega_{m} i+R_{s s}(0)
$$

where $p$ is taken to be sufficiently large to ensure $R_{s s}\left(p_{1}\right) \approx 0$ for all $p_{1}>p$. Following steps that give rise to (35) produces the sum

$$
\sum_{m=0}^{L-1} W^{2}(m)=2 L \sum_{i=1}^{p-1} R_{s s}^{2}(i)+L R_{s s}^{2}(0)
$$

Similarly, the numerator of the DR is

$$
\sum_{m=0}^{L-1} W(m) \bar{G}_{s s}(m)=2 L \sum_{i=1}^{L-1}(L-i) R_{s s}^{2}(i)+L^{2} R_{s s}^{2}(0)
$$

Putting (39) and (40) into (21) results in

$$
\mathrm{DR}_{d}=\frac{N\left[2 \sum_{i=1}^{L-1}(L-i) R_{s s}^{2}(i)+L R_{s s}^{2}(0)\right]^{2}}{2 L^{2} \sigma_{\phi}^{4}\left[2 \sum_{i=1}^{p-1} R_{s s}^{2}(i)+R_{s s}^{2}(0)\right]}
$$




\section{EXAMPLES AND SIMULATION RESULTS}

\section{Example(i)}

$s(n)$ is an ideal bandpass process of bandwidth $2 \pi \Delta$ and center frequency $\omega_{0}=0.5 \pi$. Then

$$
R_{s s}(i)=2 \Delta \frac{\sin \pi \Delta i}{\pi \Delta i} \cos \omega_{0} i
$$

Table I and II give the three DRs, for $\Lambda_{o}, \Lambda_{d}$ and $\Lambda_{a}$ as a function of $L$ and $\sigma_{\phi}^{2}=1$. Table $\mathrm{I}$ is for bandwidth $=\frac{\pi}{4}$, i.e., $\Delta=\frac{1}{8}$ and for Table II, $\Delta=\frac{1}{16}$. They show that for $\Lambda_{o}$ and $\Lambda_{d}$, their DRs increase with $L$ so that $L=N$ is always the optimal, i.e., using just the periodogram will give the optimum performance although the DR for $\Lambda_{o}$ is always greater than that of $\Lambda_{d}$. For $\Lambda_{a}$ there is an $L<N$ that gives the largest DR. To investigate this result further, Table III lists the DR for $\Lambda_{a}$ with $\Delta$ as a parameter. It clearly shows that the optimum $L$ is inversely proportional to the signal bandwidth $2 \pi \Delta$. To compare the detection performance of the three detectors, simulation of 10,000 runs was performed to produce $P_{D}$ versus $P_{F A}$, or receivers operating characteristic (ROC) curves. The simulation parameters are $N=256$, and SNR $=10 \log \frac{R_{s s}(0)}{\sigma_{\phi}^{2}}$ $=0 \mathrm{~dB}$. The simulation procedure follows that of [7]. Figure 1 contains the ROC curves for $\Lambda_{o}$, and $\Lambda_{a}$. To confirm that for $\Lambda_{a}$, the optimum $L$ is indeed inversely proportional to bandwidth, Figure 2 plots the ROC curves for $\Lambda_{a}$ at $\Delta=\frac{1}{8}$ as a function of $L$ while Figure 3 is for $\Delta=\frac{1}{16}$. From Table III, the optimum L for $\Delta=\frac{1}{8}$ is $L=8$ and for $\Delta=\frac{1}{16}$ is $L=16$. These values of $L$ indeed give the best performance in Figures 2 and 3 respectively.

TABLE I

DRS FOR $\Delta=\frac{1}{8}$

\begin{tabular}{|c||c|c|c|c|c|c|}
\hline \multicolumn{6}{|c|}{ Number of points $L$ in a Bartlett segment } \\
\hline & 8 & 16 & 32 & 64 & 128 & 256 \\
\hline$\Lambda_{o}$ & 17.01 & 22.26 & 26.01 & 28.44 & 29.94 & 30.85 \\
\hline$\Lambda_{d}$ & 14.23 & 20.36 & 24.85 & 27.79 & 29.60 & 30.66 \\
\hline$\Lambda_{a}$ & 9.84 & 6.60 & 3.63 & 1.91 & 0.98 & 0.50 \\
\hline
\end{tabular}

TABLE II

DRS FOR $\Delta=\frac{1}{16}$

\begin{tabular}{|c||c|c|c|c|c|c|}
\hline \multicolumn{7}{|c|}{ Number of points $L$ in a Bartlett segment } \\
\hline & 8 & 16 & 32 & 64 & 128 & 256 \\
\hline$\Lambda_{o}$ & 5.10 & 8.25 & 11.01 & 12.94 & 14.19 & 14.96 \\
\hline$\Lambda_{d}$ & 3.14 & 6.95 & 10.08 & 12.38 & 13.87 & 14.79 \\
\hline$\Lambda_{a}$ & 3.53 & 4.82 & 3.27 & 1.81 & 0.95 & 0.49 \\
\hline
\end{tabular}

TABLE III

DRS FOR $\Lambda_{a}$

\begin{tabular}{|c||c|c|c|c|c|c|c|}
\hline \multicolumn{1}{|c||}{} & \multicolumn{7}{c|}{ Number of points $L$ in a Bartlett segment } \\
\hline$\Delta$ & 4 & 8 & 16 & 32 & 64 & 128 & 256 \\
\hline $1 / 32$ & 0.50 & 0.97 & 1.75 & 2.40 & 1.63 & 0.91 & 0.48 \\
\hline $1 / 16$ & 1.95 & 3.53 & 4.82 & 3.27 & 1.81 & 0.95 & 0.49 \\
\hline $1 / 8$ & 7.23 & 9.84 & 6.60 & 3.63 & 1.91 & 0.98 & 0.495 \\
\hline $1 / 4$ & 21.43 & 13.68 & 7.39 & 3.85 & 1.96 & 0.99 & 0.498 \\
\hline
\end{tabular}

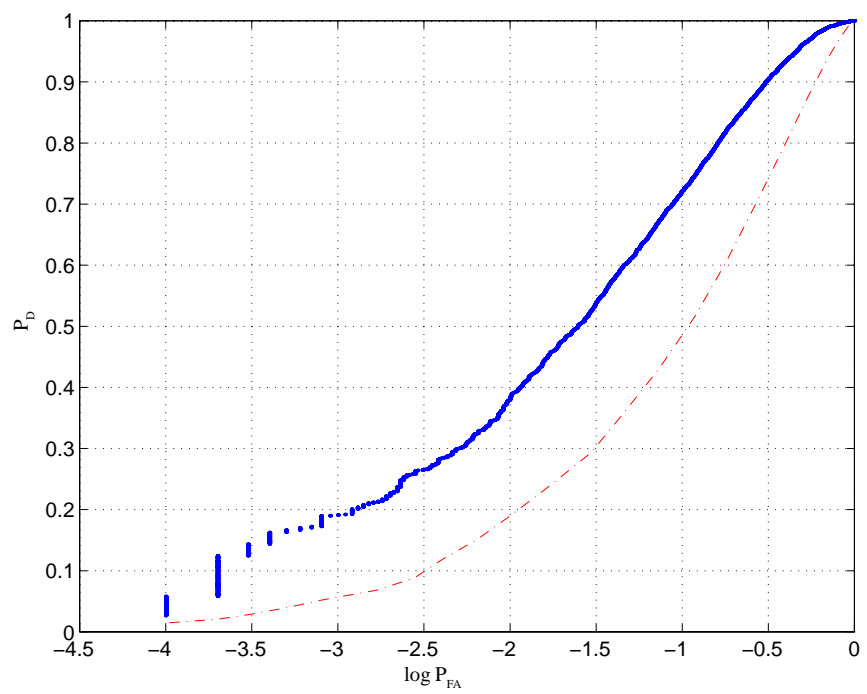

Fig. 1. ROC curves for the maximum detection of $\Lambda_{a}$ (dashed line, $L=8$ points) and $\Lambda_{o}$ (solid line, $L=256$ points) with $\Delta=\frac{1}{8}$

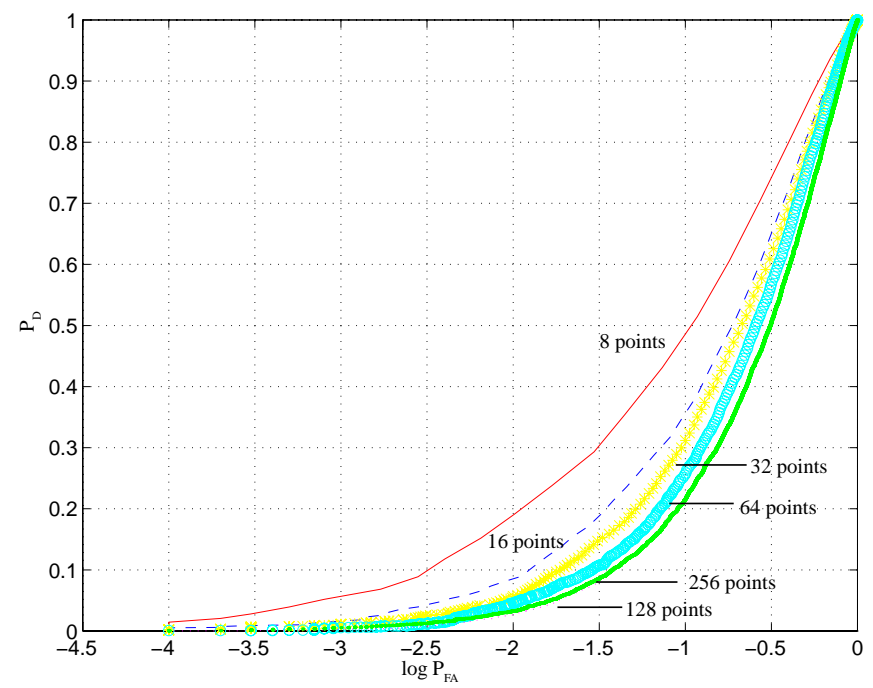

Fig. 2. ROC curves for $\Lambda_{a}$ and $\Delta=\frac{1}{8}$ 


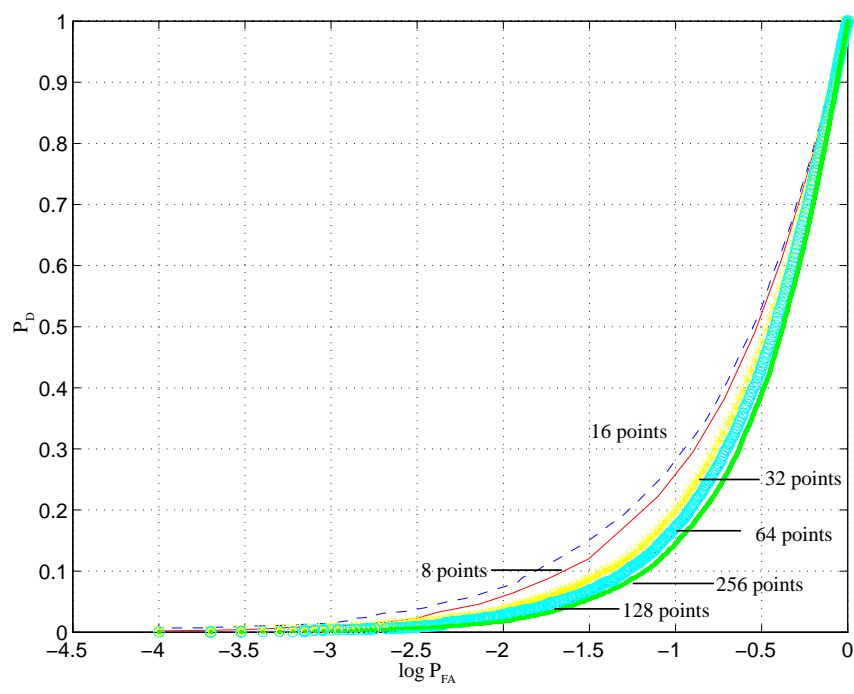

Fig. 3. ROC curves for $\Lambda_{a}$ and $\Delta=\frac{1}{16}$

\section{Example (ii)}

$s(n)$ is a narrowband process centered at $\omega_{0}$ with autocorrelation

$$
R_{s s}(i)=\sigma_{s}^{2} \rho^{|i|} \cos \omega_{0} i
$$

Again the DRs of $\Lambda_{o}, \Lambda_{d}$ and $\Lambda_{a}$ are computed as a function of $L$ for Table IV, $\rho=0.92$ and Table V, $\rho=0.997$. The carrier frequency $\omega_{0}=0.5 \pi$ and $\sigma_{s}^{2}=\sigma_{\phi}^{2}=1$. These tables show that $\Lambda_{o}$ always has the highest DR, confirming that it is the optimal detector.

TABLE IV

DRs FOR $\Lambda_{o}, \Lambda_{d}$ AND $\Lambda_{a}, \rho=0.92$

\begin{tabular}{|c||c|c|c|c|}
\hline \multicolumn{5}{|c|}{ Number of points $L$ in a Bartlett segment } \\
\hline & 8 & 16 & 32 & 128 \\
\hline$\Lambda_{o}$ & $2.6989 \times 10^{2}$ & $4.0529 \times 10^{2}$ & $0.5433 \times 10^{3}$ & $0.7068 \times 10^{3}$ \\
\hline$\Lambda_{d}$ & $1.6248 \times 10^{2}$ & $3.3499 \times 10^{2}$ & $0.5170 \times 10^{3}$ & $0.7051 \times 10^{3}$ \\
\hline$\Lambda_{a}$ & $1.7311 \times 10^{2}$ & $2.3453 \times 10^{2}$ & $2.4634 \times 10^{2}$ & $1.1881 \times 10^{2}$ \\
\hline
\end{tabular}

TABLE V

DRs FOR $\Lambda_{o}, \Lambda_{d}$ AND $\Lambda_{a}, \rho=0.997$

\begin{tabular}{|c||c|c|c|c|}
\hline \multicolumn{5}{|c|}{ Number of points $L$ in a Bartlett segment } \\
\hline & 8 & 16 & 32 & 128 \\
\hline$\Lambda_{o}$ & $3.4820 \times 10^{2}$ & $0.6722 \times 10^{3}$ & $1.3051 \times 10^{3}$ & $4.5550 \times 10^{3}$ \\
\hline$\Lambda_{d}$ & $1.1945 \times 10^{1}$ & $4.6243 \times 10^{1}$ & $1.7367 \times 10^{2}$ & $1.9477 \times 10^{3}$ \\
\hline$\Lambda_{a}$ & $2.5221 \times 10^{2}$ & $4.9620 \times 10^{2}$ & $0.8613 \times 10^{3}$ & $3.1957 \times 10^{3}$ \\
\hline
\end{tabular}

\section{CONCLUSIONS}

While there is a theoretical optimum detector in the continuous frequency domain for a signal with known PSD, its discrete implementation, which is a necessity in practice, is only an approximation. Using maximum deflection ratio as the optimum criterion, this paper has developed an optimum detector which has an exact implementation by FFT. It weights the data spectral estimate and sums the results to produce a test statistic. Computation of the optimum weights requires a complete knowledge of the signal PSD. When the PSD is not known, a simpler but sub-optimum detector $\Lambda_{a}$ results. It takes just the peak of the data spectral estimate as a test statistic. The DR of this detector is dependent on the segment length $L$ used in the spectral estimate. There is an optimum $L$, not necessarily equal to the available data length $N$, that maximizes the DR. This length $L$ is approximately inversely proportional to the signal bandwidth. Thus with only a knowledge of the signal bandwidth, it is possible to design a detector $\Lambda_{a}$ that maximizes the DR. Such a detector has important applications since in many practical situations, the signal bandwidth is approximately known.

Examples and simulation results are provided to compare the optimal discrete detector versus the approximate realization. The gain in performance is most significant at high SNR. The performance of the $\Lambda_{a}$ detector is also studied. Simulation results clearly demonstrated the importance of selecting an optimum length $L$ in forming the spectral estimate for the detector.

\section{REFERENCES}

[1] W. A. Gardner, Statistical Spectral Analysis, Prentice-Hall, Englewood Cliffs, N.J. U.S.A. 1988.

[2] H. V. Poor, An Introduction to Signal Detection and Estimation, 2nd Edition, Springer-Verlag, New York, N.Y. U.S.A. 1994.

[3] V. V. Veeravalli and H. V. Poor, "Quadratic Detection of Signal with Drifting Phase", J. Acoust. Soc. Am. Vol. 89, No. 2, pp. 811819, Feb. 1991.

[4] S. M. Kay, Modern Spectral Estimation, Prentice-Hall, Englewood Cliffs, N.J. U.S.A. 1988.

[5] L. B. W. Jolley, Summation of Series, Dover Publications Inc. New York, N.Y. U.S.A. 1961.

[6] M. Schwartz and L. Shaw, Signal Processing: Discrete Spectral Analysis, Detection and Estimation, McGraw-Hill, New York, N.Y. U.S.A. 1975.

[7] E. K. L. Hung and R. W. Herring, "Simulation Experiments to Compare the Signal Detection Properties of DFT and MEM Spectra", IEEE Trans. Acount. Speech, Signal Processing, Vol. 29, No. 5, pp. 1084-1089, Oct. 1981. 